\title{
Autologous Immunoglobulin Idiotype-KLH Conjugate Vaccine
}

National Cancer Institute

\section{Source}

National Cancer Institute. Autologous Immunoglobulin Idiotype-KLH Conjugate Vaccine. NCl Thesaurus. Code C38715.

A cancer vaccine composed of tumor-specific idiotype determinants derived from an individual's tumor cells which are conjugated to keyhole limpet hemocyanin, an immunostimulant carrier protein. When injected into the individual from whom the tumor cells were isolated, this vaccine may stimulate an antitumoral cytotoxic T-lymphocytic immune response. ( $\mathrm{NCl04)}$ 